\title{
Cross-Layer Throughput Optimization in Cognitive Radio Networks with SINR Constraints
}

\author{
Miao Ma and Danny H. K. Tsang \\ Department of Electronic and Computer Engineering, The Hong Kong University of Science and Technology, Clear Water Bay, \\ Kowloon, Hong Kong \\ Correspondence should be addressed to Miao Ma, eemma@ust.hk
}

Received 22 September 2009; Revised 10 February 2010; Accepted 14 April 2010

Academic Editor: Massimiliano Laddomada

Copyright ( $) 2010$ M. Ma and D. H. K. Tsang. This is an open access article distributed under the Creative Commons Attribution License, which permits unrestricted use, distribution, and reproduction in any medium, provided the original work is properly cited.

\begin{abstract}
Recently, there have been some research works in the design of cross-layer protocols for cognitive radio (CR) networks, where the Protocol Model is used to model the radio interference. In this paper we consider a multihop multi-channel CR network. We use a more realistic Signal-to-Interference-plus-Noise Ratio (SINR) model for radio interference and study the following cross-layer throughput optimization problem: (1) Given a set of secondary users with random but fixed location, and a set of traffic flows, what is the max-min achievable throughput? (2) To achieve the optimum, how to choose the set of active links, how to assign the channels to each active link, and how to route the flows? To the end, we present a formal mathematical formulation with the objective of maximizing the minimum end-to-end flow throughput. Since the formulation is in the forms of mixed integer nonlinear programming (MINLP), which is generally a hard problem, we develop a heuristic method by solving a relaxation of the original problem, followed by rounding and simple local optimization. Simulation results show that the heuristic approach performs very well, that is, the solutions obtained by the heuristic are very close to the global optimum obtained via LINGO.
\end{abstract}

\section{Introduction}

Cognitive radio technology [1-3] provides a novel way to solve the spectrum underutilization problem. In cognitive radio $(\mathrm{CR})$ networks, there are two types of users: primary users and secondary users. A primary user is the rightful owner of a channel, while a secondary user periodically scans the channels, identifies the currently unused channels, and accesses the channels opportunistically. The secondary users organize among themselves an ad hoc network and communicate with each other using these identified available channels. As a result, a multihop multichannel CR network is formed. How to efficiently share the spectrum holes among the secondary users, therefore, is of interest.

In this paper, we are interested in studying the opportunistic spectrum sharing problem among the secondary users, but our concern is on a cross-layer design of spectrum sharing and routing with SINR constraints. The main issues we are going to address include the following.
(1) Given a set of secondary users with random but fixed location, and a set of traffic flows, what is the max-min achievable throughput?

(2) To achieve the optimum, how to choose the set of active links, how to assign the channels to each active link, and how to route the flows?

There have been some research works on cross-layer protocols in CR networks. Hou et al. [4] characterized the behaviors and constraints for a cognitive radio network from multiple layers, including modeling the spectrum sharing and subband division, scheduling and interference constraints, and flow routing. Shi and Hou [5] developed a formal mathematical model for scheduling feasibility under the influence of power control; the formulation is a crosslayer design optimization problem encompassing power control, scheduling, and flow routing. Subsequently, on the basis of the work in [5], Shi and Hou [6] implemented their cross-layer optimization framework in a distributed manner and compared the performance of the distributed 
optimization algorithm with the upper bound and validated the efficacy. The work in [4-6] assume that the links are unidirectional, and to avoid collision only the designated receiver is need to be out of the interference caused by another transmitter. Ma and Tsang [7] proposed a crosslayer design on spectrum sharing and power control, where bidirectional links were considered and all nodes were operated at an optimal common power level at which the total spectrum utilization is maximized. Ma and Tsang [8] also proposed a cross-layer design on spectrum sharing and routing, where the channel heterogeneity (which is a unique feature for cognitive radio) was considered and modeled.

In the previous work, however, a common limitation exists since all such cross-layer protocols [4-8] are designed on the basis of the Protocol Model for radio interference, where the interference range is assumed to be limited and no interference is caused beyond the interference range. As a result, in the Protocol Model the conflict relationships among the wireless links are binary. However, in reality the aggregate interference of a large number of far transmitters could be significant and may cause interference on a receiver, and a near transmitter may not necessarily cause interference on a receiver if the transmitter properly controls its transmission power. Therefore, a definite criticism of the Protocol Model is that interference is not a binary relationship [9-11].

In order to solve the above realistic problems, the Signalto-Interference-plus-Noise Ratio (SINR) model is adopted. The rationale of SINR model is to compare the SINR with the additive interference calculation at the receiver with a threshold. Some researchers have adopted the SINR model when they consider the link scheduling, power control, or throughput improvement and and so forth. in wireless networks. For example, Brar et al. [11] investigated throughput improvements in wireless mesh networks by replacing CSMA/CA with an STDMA scheme where transmissions were scheduled according to the SINR model. Chafekar et al. [12] studied a cross-layer latency minimization problem in wireless networks with SINR model for interference. Behzad and Rubin [13] developed a new mathematical programming formulation for minimizing the schedule length in multihop wireless networks while meeting the requirements on the SINR at intended receivers.

In this paper, we consider a multihop multi-channel CR network. We adopt the (more realistic) Signal-toInterference-plus-Noise Ratio (SINR) model to study the wireless channel interference. Different from the work in [4-6], we consider the links being bidirectional because we believe the link level acknowledgments in an ad hoc network are a must. We propose a cross-layer optimization framework which jointly considers the spectrum sharing and routing with SINR constraints. The optimization problem is in the forms of a mixed integer nonlinear programming (MINLP) and the objective is to maximize the minimum end-to-end flow throughput. Since the MINLP formulation is NP-hard in general, we present a heuristic methodology by solving a relaxation of the original problem, followed by rounding and simple local optimization. Simulation results show that the heuristic approach works very well; that is, the solutions obtained by the heuristic are very close to the global optimum obtained via LINGO [14].

The rest of this paper is organized as follows. In Section 2, we describe the assumptions and system model. Section 3 introduces two interference models: one is protocol model and the other is SINR model. Section 4 presents the crosslayer design of spectrum sharing and routing with SINR constraints, and the formulation is in the forms of a mixed integer nonlinear programming (MINLP) problem. The heuristic approach is proposed in Section 5 to solve the MINLP problem. Section 6 presents the simulation results. Finally, Section 7 concludes the paper.

\section{Assumptions and System Model}

We consider a cognitive radio (CR) network with $n$ secondary users, denoted by the set $V$ and the cardinality $|V|=n$. There are $M$ orthogonal channels in the network, denoted by the set $C$ and the cardinality $|C|=M$. Each secondary user individually detects the available channels, and the set of available channels that can be used for communication is different from node to node. Let $C_{i}$ denote the set of available channels observed by node $i$, and we have $C_{i} \subseteq C$.

Each secondary user $i$ (where $1 \leq i \leq n$ ) has a programmable number of radio interfaces, denoted by $\gamma_{i}$. We assume that the radio interface is able to tune in a wide range of channels, but at a specific time each radio interface can only operate on one channel [15].

2.1. Static Node Location with a Centralized Server. We assume that the node locations are static. We also assume the set of available channel at each secondary user is static. This corresponds to the applications with a slow varying spectrum environment (e.g., TV broadcast bands). We assume that there exists a centralized server in the CR network. Each secondary user reports its location and the set of available channels to the spectrum server. The spectrum management and flow routing, therefore, is simple and coordinated. Note that the formulations in the work $[4,5,8]$ are also centralized and for static scenario (i.e., both node location and set of available channels at each node are static). Table 1 lists the notations used in this paper.

2.2. Bidirectional Links. We consider bidirectional links, rather than unidirectional links, due to two reasons [16].

(1) Wireless medium is lossy. We cannot assume that a packet can be successfully received by a neighbor unless the neighbor acknowledges it. In an ad hoc network, the link level acknowledgments are necessary.

(2) Medium access controls such as IEEE 802.11 implicitly rely on bi-directionality assumptions. For example, a RTS-CTS exchange is usually used to perform virtual carrier sensing.

Thus, if node $i$ can transmit data to node $j$ and vice versa, then we represent this by a (bidirectional) link, denoted by 
TABLe 1: Notations.

\begin{tabular}{|c|c|}
\hline Symbol & Meaning \\
\hline$V$ & Set of secondary users \\
\hline$n$ & Number of secondary users $|V|$ \\
\hline E & Set of potential links \\
\hline G & Network graph \\
\hline C & Set of available channels \\
\hline$M$ & Number of available channels $|C|$ \\
\hline$E_{i}$ & Set of links incident on node $i$ \\
\hline$C_{i}$ & Set of available channels at node $i$ \\
\hline$C_{e}$ & Set of available channels at link $e$ \\
\hline$\gamma_{i}$ & Number of radio interfaces at node $i$ \\
\hline$r_{i}$ & Transmission range for node $i$ \\
\hline$R_{i}$ & Interference range for node $i$ \\
\hline$\Delta$ & Guard zone \\
\hline$E^{\prime}$ & $\begin{array}{l}\text { Set of links that have simultaneous transmissions with } \\
\text { link } e \text { by using a same channel }\end{array}$ \\
\hline$E_{m}^{\prime}$ & $\begin{array}{l}\text { Set of links that have simultaneous transmissions with } \\
\text { link } e \text { by using channel } m\end{array}$ \\
\hline$P$ & Common transmission power \\
\hline$N_{i}$ & Noise power at node $i$ \\
\hline$I_{i}$ & Interference power at node $i$ \\
\hline$N$ & Noise power at each node \\
\hline$\beta$ & $\begin{array}{l}\text { The minimum threshold to decode a transmission at } \\
\text { a receiver }\end{array}$ \\
\hline$d_{i j}$ & Distance between nodes $i$ and $j$ \\
\hline$G_{i j}$ & Propagation gain from node $i$ to node $j$ \\
\hline$\eta$ & Path loss exponent \\
\hline$x_{e}^{m}$ & $\begin{array}{l}\text { Binary variable indicating if link } e \text { is active on channel } \\
m\end{array}$ \\
\hline$B_{e}^{m}$ & Capacity of link $e$ by using channel $m$ \\
\hline$T$ & Minimum end-to-end flow throughput \\
\hline$W$ & Bandwidth of a channel \\
\hline Q & Number of communication sessions \\
\hline$s(q)$ & Source node for session $q$ \\
\hline$d(q)$ & Destination node for session $q$ \\
\hline$f_{i, j}^{q}$ & Traffic flow from $i$ to $j$ for session $q$ \\
\hline$f_{j, i}^{q}$ & Traffic flow from $j$ to $i$ for session $q$ \\
\hline
\end{tabular}

$e=(i, j)$, between node $i$ and node $j$. Moreover, we let $C_{e}$ denote the set of available channels for the link $e$, and we have $C_{e}=C_{i} \cap C_{j}$.

2.3. Common Transmission Power. According to the study by Narayanaswamy et al. [16], to ensure that links are bidirectional, the simplest approach is to assume that nodes are homogeneous; that is, nodes transmit at the same power. In this paper we assume that each secondary user is equipped with an omnidirectional antenna. Similar to [16], we also assume that each secondary user transmits at the same power. Note that this assumption is used in [4] as well.

A bidirectional link, denoted by $e=(i, j)$, can be established between nodes $i$ and $j$ if there exists a transmission power $P$ under which the Signal-to-Noise Ratio (SNR) in the absence of cochannel interference at nodes $i$ and $j$ is not less than a threshold $\beta$, that is,

$$
\begin{gathered}
\frac{P \cdot G_{i j}}{N_{j}} \geq \beta, \\
\frac{P \cdot G_{j i}}{N_{i}} \geq \beta,
\end{gathered}
$$

where $\beta$ is signal-to-noise Ratio (SNR) threshold, $G_{i j}$ (and $G_{j i}$ ) denotes the channel propagation gain from $i$ to $j$ (and from $j$ to $i$ ), and $N_{j}$ (and $N_{i}$ ) denotes the noise power at node $j$ (and node $i$ ). Since it has been commonly assumed that $G_{i j}$ is equal to $G_{j i}[4-8,17-21]$, and $N_{i}$ is equal to $N_{j}$, we make the same assumptions here and thus we have

$$
\frac{P \cdot G_{i j}}{N} \geq \beta
$$

We let $E_{i}$ denote the set of links incident on node $i$, which can be obtained by

$$
E_{i}=\left\{e: \frac{P \cdot G_{i j}}{N} \geq \beta\right\} .
$$

Let $E$ denote the union of $E_{i}$; we have

$$
E=\bigcup_{i \in V} E_{i}
$$

As a result, we obtain an undirected connectivity graph $G=(V, E)$ to represent the CR network, where $V$ is the set of secondary users denoted by the vertices of the graph, and $E$ is the set of edges between two vertices (i.e., secondary users).

\section{The Interference Model}

In wireless networks, there are three types of interference: duplexing interference, primary interference, and secondary interference. In this paper, we assume that links using different channels do not interfere with each other. Interference only occurs among the links sharing the same channel.

The duplexing interference constraint [22] only prohibits any node from simultaneously transmitting and receiving on any frequency band (i.e., the case in Figure 1 is not allowed).

The primary interference constraint prohibits any node from simultaneously transmitting or receiving on any band (i.e., neither case in Figures 2(a), 2(b) nor 2(c) is allowed). In other words, links that shared a common node cannot transmit or receive simultaneously on any channel. Obviously, the duplexing constraint is less stringent than the primary interference constraint. And also, the duplexing and primary interference constraints are applicable to the links which share a common node (see Figures 1 and 2), and particularly, these constraints hold irrespective of the interference model.

The secondary interference constraint, on the other hand, further prohibits any node from transmitting when a neighbor node within its interference range is receiving from another node. Different from the duplexing and primary interference constraints, the secondary interference 


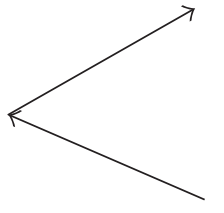

FIgURE 1: Duplexing interference constraint.

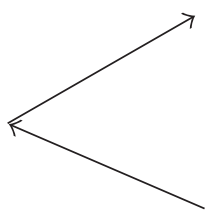

(a)

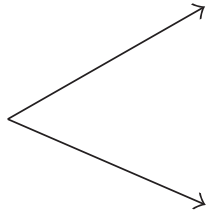

(b)

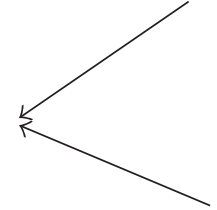

(c)
FIGURE 2: Primary interference constraint.

constraints are applicable to those links which do not share a common node (see Figure 8 shown in the appendix for better understanding).

For the purpose of modeling the secondary interference, there are two models $[23,24]$ : the Protocol Model and the Physical Model. Since rationale of the Physical Model is based on the SINR at the receiver, we call the Physical Model as the SINR model hereinafter in this paper. The relationship of these three types of interferences is shown in Figure 3.

3.1. The Protocol Model. Gupta and Kumar proposed the Protocol Model [23] which implicitly assumed that links are unidirectional. With this assumption, collisions only occur when the designated receiver is interfered by another transmitter. Basically, the Protocol Model assumes that the interference range is limited and no interference will be caused beyond the interference range. We let $r_{i}$ and $R_{i}$ denote the transmission range and interference range for any node $i$, respectively; then we have $R_{i}=(1+\Delta) r_{i}$, where $\Delta$ is the guard zone to prevent a neighboring node from transmitting on the same channel at the same time [23]. The Protocol Model claims that a transmission from node $i$ to node $j$ is successful if and only if any node $k$ which may cause interference on node $j$ (i.e., if $d_{k j} \leq R_{k}$ where $d_{k j}$ denotes the distance between $k$ and $j$ ) is not simultaneously transmitting.

A more realistic version, however, assumes that IEEE 802.11 MAC is employed and thus the links are bidirectional (due to RTS-CTS and ACK exchange). We usually call this version as 802.11-style Protocol Model [7, 8]. Suppose that link $e=(i, j)$ and link $e^{\prime}=(k, h)$ are established and both are bidirectional. They are also active on a same channel. The 802.11-style Protocol Model states that a transmission on link $e$ between nodes $i$ and $j$ is successful if and only if for any link $e^{\prime}=(k, h)$, such that $d_{k j} \leq R_{k}$ or $d_{k i} \leq R_{k}$ or $d_{h j} \leq R_{h}$ or $d_{h i} \leq R_{h}$, is not simultaneously transmitting.

Note that the Protocol Model leads to binary conflict relationships among the wireless links. In other words, any two links either interfere with each other or can be active simultaneously, regardless of the other ongoing signal transmissions.

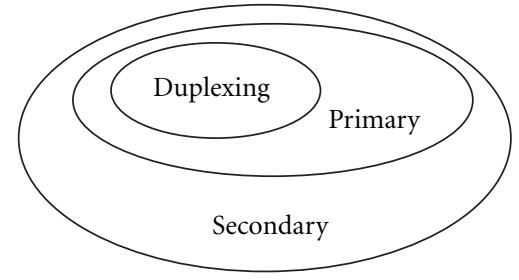

Figure 3: Relationship of three types of interferences.

3.2. The SINR Model. As we mentioned before, in reality the aggregate interference of a large number of far transmitters could be significant and may cause interference at the receiver, and a near transmitter may not necessarily cause interference at the receiver if the transmitter properly controls its transmission power. Thus, the main limitation of the Protocol Model is that interference is not a binary relationship. These problems can be overcome by means of the SINR model, whose rationale is as follows.

3.2.1. Unidirectional Links. Before we consider bidirectional link, let us first consider unidirectional link. For clarification, we let $e=i \rightarrow j$ and $e^{\prime}=k \rightarrow h$ denote two unidirectional links and suppose that they are active on a same channel. The transmission from node $i$ is successfully received by node $j$ if and only if the SINR at the receiving node $j$ is not less than a threshold $\beta$. That is,

$$
\frac{P \cdot G_{i j}}{I_{j}+N} \geq \beta,
$$

where $I_{j}$ denotes the interference power at node $j$. To calculate $I_{j}$, we need to sum all the links $\left\{e^{\prime}=k \rightarrow h\right\}$ that have simultaneous transmissions with link $e$ on a same channel. Therefore we have

$$
\frac{P \cdot G_{i j}}{P \cdot \sum_{k \rightarrow h \in E^{\prime}} G_{k j}+N} \geq \beta,
$$

where $E^{\prime}$ contains all links that have transmissions concurrent with link $e$ by using a same channel. The SINR model accurately captures the fact that interference is caused by aggregate effect of the simultaneous active links.

3.2.2. Bidirectional Links. Next, we extend the SINR model from unidirectional link to bidirectional link. To distinguish from the unidirectional links, we let $e=(i, j)$ and $e^{\prime}=(k, h)$ denote two bidirectional links and suppose that they are active on a same channel. Because the interference raised by node $k$ and node $h$ might be different, we need to choose the maximum one. To ensure the transmission on link $e$ between nodes $i$ and $j$ to be successful, the SINR at both nodes $i$ and $j$ is not less than a threshold $\beta$. We also need to sum all the links $\left\{e^{\prime}=(k, h)\right\}$ that have simultaneous transmissions with link $e$ on a same channel. To the end, we obtain

$$
\frac{P \cdot G_{i j}}{P \cdot \sum_{(k, h) \in E^{\prime}} \max \left(G_{k j}, G_{h j}, G_{k i}, G_{h i}\right)+N} \geq \beta .
$$


Note that the SINR model is more accurate than the Protocol Model since it better captures the physical propagation. Moreover, in the SINR model a correct packet reception is allowed even in the presence of (moderate) interference, and the cumulative character of interference is taken into account. The main drawback of this model lies in its high complexity, as the interference is described as the complex mathematical relationships.

\section{Cross-Layer Design of Spectrum Sharing and Routing}

In this section, we present a cross-layer optimization framework which jointly designs the spectrum sharing and routing. Spectrum sharing can be done either in time domain or in frequency domain. In this paper, we consider frequency domain channel assignment. Spectrum sharing is to determine which link is going to be active and which channel will be assigned to each active link, and our target is to form a conflict-free topology. Routing is to determine which path each traffic flow is going to travel from the source node to the destination node. We allow multipath for each traffic flow. Different from the previous work, in this paper, we adopt the SINR model for radio interference and consider links being bidirectional.

4.1. Link Assignment. We say that link $e$ is active only if there is a transmission on channel $m$ over link $e$. We define a $0-1$ binary variable $x_{e}^{m}$ as follows:

$$
x_{e}^{m}= \begin{cases}1 & \text { if link } e \text { is active on channel } m, \\ 0 & \text { otherwise }\end{cases}
$$

4.2. Interference Constraints. In this paper, we consider both primary and secondary interference constraints. We term the secondary interference constraints as the SINR constraints hereinafter in this paper, since we use the SINR model to model them.

In the remaining part of this subsection, we let $e=(i, j)$ denote a link and let $e^{\prime}=(k, h)$ denote another link. Both links are active and use a same channel $m$ for transmission.

4.2.1. Primary Interference Constraints. By using a same channel, each node can either transmit or receive but not both, at a given time. In other words, links that share a common node cannot transmit or receive simultaneously on any channel.

For ease of presentation and also for notational convenience, each link is also understood as a set of two nodes; then we define

$$
e \cap e^{\prime} \begin{cases}=\varnothing & \text { if } i \neq k \text { and } j \neq k \text { and } i \neq h \text { and } j \neq h, \\ \neq \varnothing & \text { if } i=k \text { or } j=k \text { or } i=h \text { or } j=h .\end{cases}
$$

Clearly, we use the notation $e \cap e^{\prime}=\varnothing$ to denote that the two links $e$ and $e^{\prime}$ do not share a common node, and the notation $e \cap e^{\prime} \neq \varnothing$ to denote that the two links $e$ and $e^{\prime}$ share a common node.
Thus, the primary interference constraint can be expressed as follows:

$$
\begin{gathered}
x_{e}^{m}+x_{e^{\prime}}^{m} \leq 1 \\
\left(m \in C_{e} \cap C_{e^{\prime}}, e^{\prime} \cap e \neq \varnothing, e^{\prime} \neq e, e^{\prime} \in E, e \in E\right) .
\end{gathered}
$$

4.2.2. SINR (i.e., Secondary Interference) Constraints. For the links that do not share a common node but share a common channel, they are applicable to the SINR constraints if the links are active simultaneously. That is, a transmission on a bidirectional link $e=(i, j)$ is successful if and only if the SINR at either node $i$ or node $j$ is not less than the minimum required threshold $\beta$. This leads to the following constraint:

$$
\begin{aligned}
& P G_{i j} x_{e}^{m}-\beta P \sum_{e^{\prime} \in E_{m}^{\prime}} \max \left(G_{k j}, G_{h j}, G_{k i}, G_{h i}\right) x_{e^{\prime}}^{m}-\beta N \\
& \quad \geq \Upsilon\left(x_{e}^{m}-1\right) \quad\left(m \in C_{e} \cap C_{e^{\prime}}, e \cap e^{\prime}=\varnothing, e^{\prime} \in E, e \in E\right),
\end{aligned}
$$

where $E_{m}^{\prime}$ contains all links that have transmissions concurrent with link $e$ by using channel $m$. Similar to [13], we introduce a sufficiently large positive number $\Upsilon$ in the constraint (11), where the constraint becomes "redundant" when link $e$ is not active (i.e., $x_{e}^{m}=0$ ). Notice that we only sum the interference caused by those active links because link $e^{\prime}$ will not cause any interference on link $e$ whenever link $e^{\prime}$ is not active (i.e., $x_{e^{\prime}}^{m}=0$ ). And also note that when we calculate the interference caused by the active link $e^{\prime}=(k, h)$, we choose the maximum interference caused from either node $k$ or node $h$ to either node $i$ or node $j$ (due to bidirectional link).

4.3. Node-Radio Constraint. A node can establish multiple links with its neighboring nodes if it can tune each of its radio interface to a different channel. However, the number of established links at each node is constrained by the number of its radio interfaces. This leads to the following constraint:

$$
\sum_{e \in E_{i}} \sum_{m \in C_{e}} x_{e}^{m} \leq \gamma_{i} \quad(i \in V)
$$

4.4. Multipath Routing Constraints. We consider multiple traffic flows in the network. We term the traffic flow for each source-destination pair as a communication session and use $q(q=1,2, \ldots, Q)$ to index each session. Let $s(q)$ and $d(q)$ represent the source node and destination node for session $q$. Because the links are bidirectional, the traffic flow on each link can be in either direction. Thus, for any link $e=(i, j)$, we let $f_{i, j}^{q}$ (and $f_{j, i}^{q}$ ) denote the traffic flow traveling from $i$ to $j$ (and from $j$ to $i$ ) for the session $q$, where $(i, j) \in E, i \neq j$. For each traffic flow, we allow multi-path routing.

Our definition of the maximum throughput is maxmin flow rate [25]. That is, our target is to maximize the minimum end-to-end flow throughput that can be achieved 
in the network. Therefore, the multi-path routing constraints are listed as follows:

$$
\begin{gathered}
f_{i, j}^{q} \geq 0, \quad f_{j, i}^{q} \geq 0 \quad((i, j) \in E, q \in Q), \\
\sum_{\substack{k: k \neq d(q),(k, i) \in E_{i}}} f_{k, i}^{q}=\sum_{\substack{j: j \neq s(q),(i, j) \in E_{i}}} f_{i, j}^{q} \\
(i \neq d(q), i \neq s(q), i \in V, q \in Q), \\
\sum_{j:(i, j) \in E_{i}} f_{i, j}^{q} \geq T \quad(i=s(q), q \in Q), \\
\sum_{j:(j, i) \in E_{i}} f_{j, i}^{q} \geq T \quad(i=d(q), q \in Q), \\
\sum_{q \in Q} f_{i, j}^{q}+\sum_{q \in Q} f_{j, i}^{q} \leq \sum_{m \in C_{e}} B_{e}^{m} \quad(e=(i, j), e \in E),
\end{gathered}
$$

where $T$ is the minimum end-to-end throughput for every session.

The constraint (13) restricts the amount of flow on each link to be nonnegative. The constraint (14) states that at each node, except the source node and destination node, the amount of incoming flow is equal to the amount of outgoing flow. The constraint (15) represents that the minimum outgoing flow from each source node is at least $T$. The constraint (16) states that the minimum incoming flow to the destination node is at least $T$. The constraint (17) indicates that the sum of the flows over all sessions traversing a link cannot exceed the link capacity.

To calculate the link capacity, we let $W$ denote the bandwidth of each channel, and let $B_{e}^{m}$ denote the capacity of link $e$ by using channel $m$. Assuming Gaussian noise and interference, we have

$$
\begin{array}{r}
B_{e}^{m} \\
=W \log _{2}\left(1+\frac{P G_{j i} x_{e}^{m}}{P \cdot \sum_{e^{\prime} \in E_{m}^{\prime}} \max \left(G_{k j}, G_{h j}, G_{k i}, G_{h i}\right) x_{e^{\prime}}^{m}+N}\right) \\
\left(m \in C_{e} \cap C_{e^{\prime}}, e \cap e^{\prime}=\varnothing, e^{\prime} \in E, e \in E\right) .
\end{array}
$$

4.5. Problem Formulation. We aim to maximize the minimum end-to-end throughput, and this optimization problem can be formulated as

$$
\begin{gathered}
\max T \\
\text { Subject to: } x_{e}^{m}=0,1 \quad\left(m \in C_{e}, e \in E\right), \\
x_{e}^{m}+x_{e^{\prime}}^{m} \leq 1 \quad\left(m \in C_{e} \cap C_{e^{\prime}}, e^{\prime} \cap e \neq \varnothing, e^{\prime} \neq e, e^{\prime} \in E, e \in E\right), \\
P G_{i j} x_{e}^{m}-\beta P \sum_{e^{\prime} \in E_{m}^{\prime}} \max \left(G_{k j}, G_{h j}, G_{k i}, G_{h i}\right) x_{e^{\prime}}^{\mathrm{m}}-\beta N \\
\geq \Upsilon\left(x_{e}^{m}-1\right) \quad\left(m \in C_{e} \cap C_{e^{\prime}}, e \cap e^{\prime}=\varnothing, e^{\prime} \in E, e \in E\right),
\end{gathered}
$$

$$
\begin{gathered}
\sum_{e \in E_{i}} \sum_{m \in C_{e}} x_{e}^{m} \leq \gamma_{i} \quad(i \in V), \\
f_{i, j}^{q} \geq 0, \quad f_{j, i}^{q} \geq 0 \quad((i, j) \in E, q \in Q), \\
\sum_{\substack{k: k \neq d(q),(k, i) \in E_{i}}} f_{k, i}^{q}=\sum_{\substack{j: j \neq s(q),(i, j) \in E_{i}}} f_{i, j}^{q} \\
(i \neq d(q), i \neq s(q), i \in V, q \in Q), \\
\sum_{j:(i, j) \in E_{i}} f_{i, j}^{q} \geq T \quad(i=s(q), q \in Q), \\
\sum_{j:(j, i) \in E_{i}} f_{j, i}^{q} \geq T \quad(i=d(q), q \in Q), \\
\sum_{q \in Q} f_{i, j}^{q}+\sum_{q \in Q} f_{j, i}^{q} \leq \sum_{m \in C_{e}} B_{e}^{m} \quad(e=(i, j), e \in E),
\end{gathered}
$$

where $\gamma_{i}$ is constant and $B_{e}^{m}$ can be obtained by (18). $x_{e}^{m}$ (binary integer) and $f_{i, j}^{q}$ and $f_{j, i}^{q}$ (rational number) are decision variables. The objective function is a linear function; however, (28) is a nonlinear constraint. The optimization problem is in the form of mixed integer nonlinear programming (MINLP) problem and can be solved by LINGO.

\section{Finding the Optimal Solution by Heuristic}

We are interested in finding the optimal solution under which the minimum end-to-end flow throughput is maximized. However, the original problem is in the form of MINLP since the constraint (28) is nonlinear due to the logarithm function. But if we investigate the formulation more carefully, we find that the complexity of the MINLP problem formulation does not lie in the nonlinear logarithm function in constraint (28). Instead, the complexity comes from the existence of the binary variable $x_{e}^{m}$. The reason is that as long as the values of $x_{e}^{m}$ are determined (i.e., the set of active links and the channel assignment on each active link are determined), then this MINLP reduces to an LP, which can be solved in polynomial time. To this end, we develop a heuristic method by solving a relaxation of the original problem, followed by rounding and simple local optimization [26].

5.1. Relaxation. We start by relaxing the MINLP problem to the following format.

$$
\max T
$$

Subject to: $0 \leq x_{e}^{m} \leq 1 \quad\left(m \in C_{e}, e \in E\right)$

constraints (21)-(28).

That is, we allow the variables $x_{e}^{m}$ to take values between 0 and 1 . The relaxed problem can be solved in polynomial time. By solving the relaxed problem, we obtain an upper bound of the optimal value of the original problem, and we let $\mathrm{X}^{*}$ denote the relaxed solution that produces the upper bound. 
5.2. Getting Independent Sets. In order to determine the set of active links and form a conflict-free topology, we need to obtain the independent sets (i.e., the set of links that can be simultaneously active on a channel). The reason is that we can significantly speed up the search process by combining the independent sets together with the rounding and local optimization (introduced below).

To obtain the independent sets, Karnik et al. [25] proposed a smart enumerative technique. In this paper, we extend this technique into a more general case from the following two aspects. (1) In [25], all nodes are assumed to transmit at a single channel. But in our formulation we consider a more realistic scenario where each node (i.e., secondary user) is able to access a set of available channels, and especially, the set of available channels is different from node to node. (2) In [25] the links are unidirectional, but in our formulation the bidirectional links are considered.

For this technique, similar to [25] we make the following additional assumptions. (Interested readers are advised to refer [25] for details on why the above three assumptions are reasonable.)

(A1) The propagation gains are modeled by isotropic path loss. That is, the propagation gain from node $i$ to node $j$ is

$$
G_{i j}=\left(\frac{d_{0}}{d_{i j}}\right)^{\eta},
$$

where $d_{0}$ is the far-field crossover distance and $\eta$ denotes the path loss exponent.

(A2) The minimum distance for any pair of nodes is $d_{\min }$.

(A3) The nodes are located in a square size $L \times L$ area.

Theorem 1. Under the assumptions (A1)-(A3), the number of simultaneous transmissions on a same channel (i.e., the size of maximum independent sets on a channel) is upper bounded by $2 L^{2} / \pi\left(d_{\min } \beta^{1 / \eta}\right)^{2}$.

Proof. Please see the appendix.

Section 6 will show the extent of complexity reduction by using this technique together with rounding and local optimization.

5.3. Rounding. The next step is to round the relaxed problem solution $\mathbf{X}^{*}$ to a valid binary integer solution $\hat{\mathbf{X}}$. To create $\hat{\mathbf{X}}$, we can simply round the one (say $x_{e}^{m}$ ) with the largest value to 1 . According to the independent set, with $x_{e}^{m}=1$ we can immediately decide some variables which share the same channel $m$ with the link $e$ to be 1 or 0 . After fixing some decision variables to 1 or 0 in the first iteration, we update a new relaxed LP for the second iteration. We can solve this new LP, then again round the one with the largest value to 1 , and set some additional variables to 0 accordingly. The iteration continues and eventually we can determine all $\left\{x_{e}^{m}\right\}$ to either 0 or 1 .

Upon fixing all the $x_{e}^{m}$ values, the original MINLP reduces to an LP problem, which can be solved in polynomial time. It is worth emphasizing that, unlike the solutions obtained by relaxation, the final solution obtained here is a feasible solution since all $x_{e}^{m}$ values are binary instead of rational numbers.

5.4. Local Optimization. Further improvement can be obtained by a local optimization method, starting from $\hat{\mathbf{X}}$. Suppose that for channel $m$ there are $n_{m}$ independent sets, and we use $1,2, \ldots, n_{m}$ to index each independent set. Since, in the initial solution $\hat{\mathbf{X}}$, one of $n_{m}$ independent sets is active on channel $m$, then we use $v\left(1 \leq v \leq n_{m}\right)$ to index the active independent set. Then for channel $m$, we observe its independent sets and cycle through $k=1,2, \ldots,\left(n_{m}-1\right)$ while $k \neq v$, and at the $k$ th step replacing the $k$ th independent set as 1. If this change leads to an improvement for the objective function, we accept the change and continue. Otherwise we go on to the next independent set of channel $m$. We continue until we have tried all the independent sets for channel $m$. The same process repeats for all the channels. Numerical experiments show that this local optimization method can lead to significant improvement on the objective function.

\section{Simulation Results}

In this section, we present simulation results for our heuristic method and compare it with the upper bound and the global optimum. The upper bound is obtained by solving a relaxation of the original problem, while the global optimum is obtained by LINGO which is a mathematical software package. The default settings for the simulations are as follows. The noise power at every receiver is equal to $-100 \mathrm{dBm} . \eta$ and $d_{0}$ are taken to be 4 and $0.1 \mathrm{~m}$, respectively. The minimum threshold $(\beta)$ is set to $2.3 \mathrm{~dB}$.

We consider two scenarios: one is regular topology and the other is random topology. We make no claims that these topologies are representative of typical cognitive radio networks. The reason that we have chosen these two simple topologies is to facilitate detailed discussion of the results and for the illustration purpose. For the propagation model, we adopt the isotropic path loss shown in (31). However, we stress that the validity of the conclusions drawn in the following holds for any scenario and also when more complicated propagation models are used to determine $G_{i j}$ parameters.

6.1. Performance in Regular Topology. We first look at the performance of the proposed approach in the regular topology, as illustrated in Figure 4(a). A total number of $n=9$ nodes are placed in a $3 \times 3$ grid, and the deployment area is a square size of $80 \times 80$. The unit grid separation (i.e., distance between adjacent nodes along the grid-side) is $20 \mathrm{~m}$. All nodes use a common transmit power of $3 \mathrm{~mW}$, which results in a transmission range of $23.4 \mathrm{~m}$. The transmission range is greater than the unit grid separation but is less than the unit grid diagonal (i.e., distance between adjacent nodes along the diagonal). This results in a simple topology where 
TABLe 2: Set of available channels at each node (i.e., $C_{i}$ ) for regular topology.

\begin{tabular}{lcc}
\hline Node index & Location & Available channels \\
\hline 1 & $(20,20)$ & $2,3,4$ \\
2 & $(20,40)$ & $1,2,4,6$ \\
3 & $(20,60)$ & $1,2,5$, \\
4 & $(40,20)$ & $3,4,5,6$ \\
5 & $(40,40)$ & $1,3,4,6$ \\
6 & $(40,60)$ & $1,3,5$ \\
7 & $(60,20)$ & $1,2,5,6$ \\
8 & $(60,40)$ & $2,4,5$ \\
9 & $(60,60)$ & $1,2,3,6$ \\
\hline
\end{tabular}

all nodes can only communicate with their physical onehop neighbors on the grid. Figure 4(b) shows the connection graph. There are $M=6$ channels that can be used for the entire network. Every node has 3 radio interfaces $\left(\gamma_{i}\right)$. The set of available channels at each node is randomly generated; see Table 2. Note that the set of available channels is different from node to node.

6.1.1. Complexity Reduction. For this scenario, there are 12 potential links and 24 binary variables (i.e., $\left\{x_{e}^{m}\right\}$ ). By using the enumerative technique, we obtain 22 independent sets and the size of the maximum independent set is 2 . The exhaustive search space to determine the binary variable is $2^{24}$; however, combining the independent sets together with the rounding and local optimization, the search space is significantly reduced from $2^{24}$ to 1800 .

6.1.2. Throughput. Regarding the traffic flow, we consider $|Q|=1,2$, or 3 active sessions and run 3 experiments, respectively. In each experiment, the source node and destination node for each session are randomly generated. Figure 5(a) shows the results of the throughput obtained by our heuristic, upper bound and global optimum. Since the regular topology is simple, the heuristic method includes rounding technique only. It is observed that such heuristic results (obtained by rounding technique only) are equal to the global optimum, therefore no need to carry out local optimization. It is also found that there are gaps between the heuristic results and the relaxation bound.

For comparison purpose Figure 5(b) shows the results of the optimality ratio (which is defined as the normalized throughput over the global optimum) obtained by our rounding technique. It is found that the optimality ratio obtained by rounding is 1 , while the optimality ratio of the relaxation bound is within $(1,1.6)$. Simulation results show that the rounding technique performs very well in this scenario.

6.2. Performance in Random Topology. We next relax the regularity of node placement and look at the performance of the proposed approach in the random topology. As Figure 6(a) shows, we assume that $n=10$ nodes are uniformly distributed in a square size of $40 \times 40$ area. All
TABLE 3: Set of available channels at each node (i.e., $C_{i}$ ) for random topology.

\begin{tabular}{lcc}
\hline Node index & Location & Available channels \\
\hline 1 & $(29.0,28.8)$ & $1,2,6,7$ \\
2 & $(23.2,9.9)$ & $3,4,5,7$ \\
3 & $(12.4,13.7)$ & $5,7,8$ \\
4 & $(4.4,38.8)$ & $3,4,6$ \\
5 & $(15.3,2.9)$ & $1,2,3,4,7,8$ \\
6 & $(12.8,30.5)$ & 2,5 \\
7 & $(38.0,10.8)$ & $1,4,6$ \\
8 & $(1.6,6.1)$ & $3,4,5$ \\
9 & $(24.3,37.7)$ & $2,4,5,8$ \\
10 & $(39.1,34.1)$ & $1,3,6,8$ \\
\hline
\end{tabular}

nodes use a common transmit power of $4 \mathrm{~mW}$, which results in a transmission range of $25.1 \mathrm{~m}$. Figure $6(\mathrm{~b})$ shows the connection graph. There are $M=8$ channels that can be used for the entire network. Every node has 4 radio interfaces $\left(\gamma_{i}\right)$. The set of available channels at each node is shown in Table 3. Again, the set of available channels is different from node to node.

6.2.1. Complexity Reduction. For this scenario, there are 21 potential links and 32 binary variables (i.e., $\left.\left\{x_{e}^{m}\right\}\right)$. By using the enumerative technique, we get 29 independent sets and the size of the maximum independent set is 2 . The exhaustive search space to determine the binary variable is $2^{32}$; however, combining the independent sets together with the rounding and local optimization, the search space is significantly reduced from $2^{32}$ to 13500 .

6.2.2. Throughput. Regarding the traffic flow, we consider $|Q|=1,2,3,4$, or 5 active sessions and run 15 experiments, respectively. In each experiment, the source node and destination node for each session are randomly generated. Different from the results obtained in the regular topology, in this random topology we show not only the heuristic results obtained by rounding technique but also the heuristic solutions obtained by rounding and local optimization. Table 4 shows the results. It is observed that there are some minor gaps between global optimum and the heuristic results obtained by rounding technique. However, by further using local optimization method, we find that the heuristic results are very close to the the global optimum. This observation demonstrates that the local optimization can lead to significant improvement on the objective function. Also note that there are some moderate gaps between the global optimum and the bounds obtained by relaxation.

For comparison purpose Table 5 shows the optimality ratio obtained by our heuristic and relaxation. It is observed that the heuristic results obtained by rounding and local optimization are very close to 1 , while the heuristic results obtained by only rounding are within $(0.48,1.0)$ and the ratio of the relaxation is within $(1,1.8)$. The simulation results show that the combination of rounding and local optimization performs very well in this scenario. 


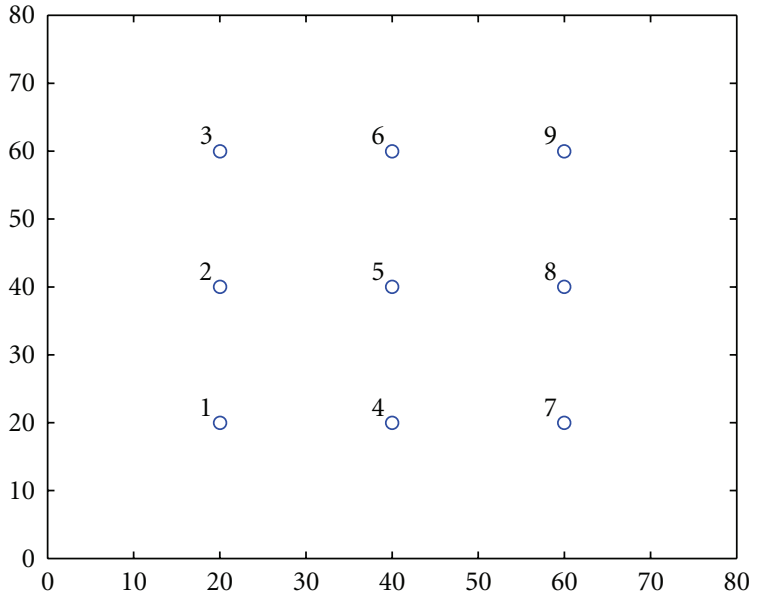

(a) Node location

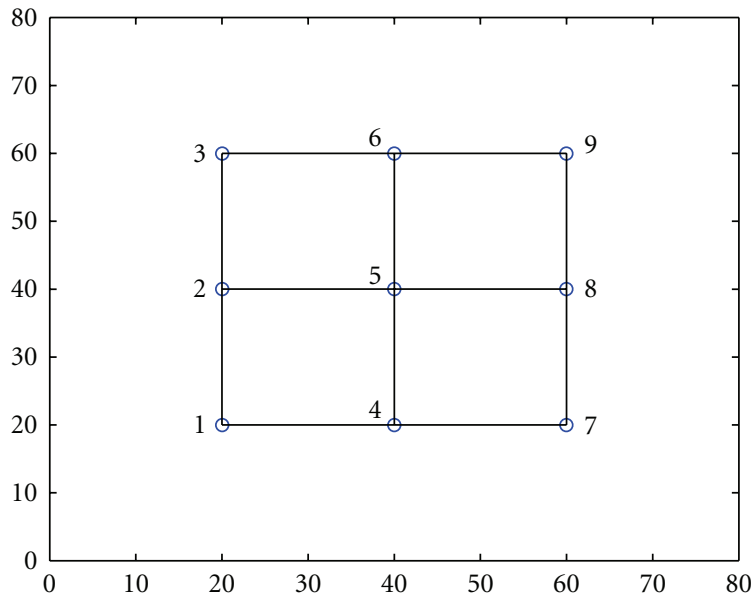

(b) Connectivity Graph

FIGURE 4: Regular topology.

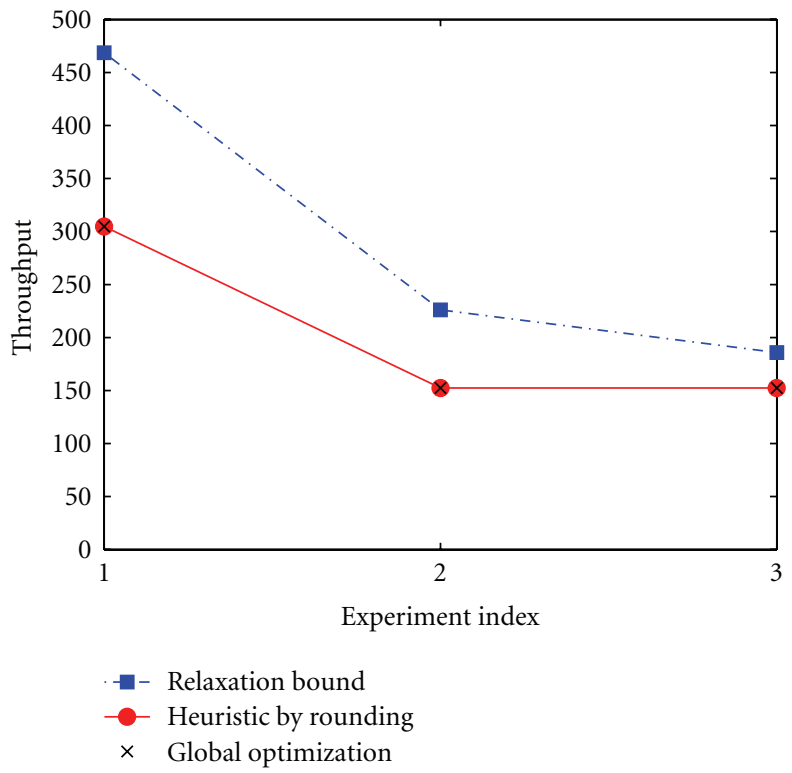

(a) Throughput

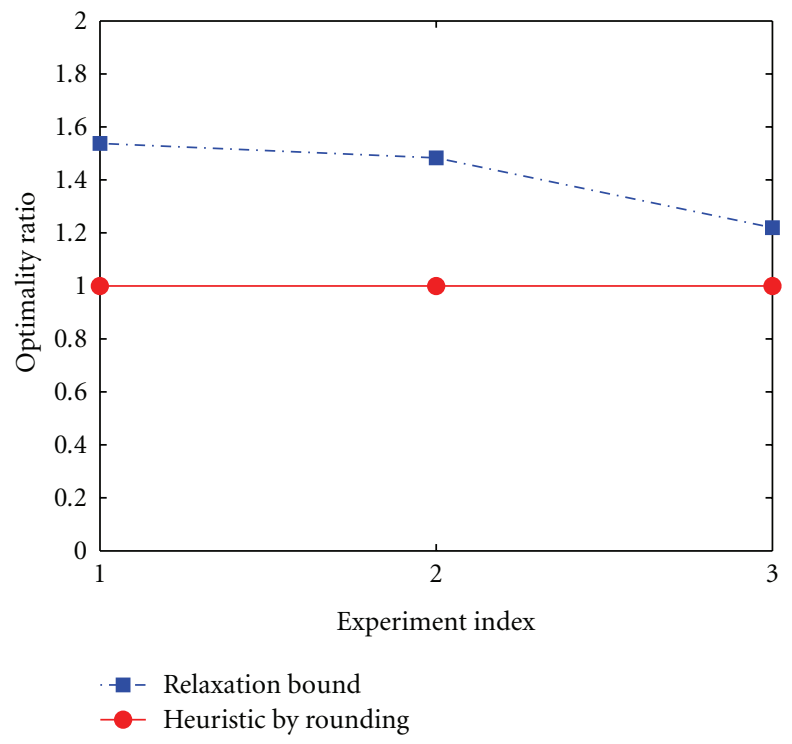

(b) Optimality ratio

FIGURE 5: Comparison between heuristic, upper bound and global optimum for regular topology.

6.2.3. Spectrum Sharing and Routing. For illustration purpose, we show the results of spectrum sharing and routing when there are 5 communication sessions. The source node and destination node for each communication session are randomly generated; see Table 6 .

By solving the MINLP problem by heuristic, we obtain that the optimal achievable throughput for each traffic flow is 111.3 (which is the 15th experiment shown in Figure 7). Figure 7(a) illustrates the optimal spectrum sharing. It is noticed that there are 10 active links in total, and channels 4 and 8 are reused. The nodes form themselves as an ad hoc network and all links can be active simultaneously (i.e., the topology is conflict-free). Figures $7(\mathrm{~b})-7$ (f) illustrate the routing path(s) for each traffic flow. Figure 7(b) shows that the traffic flow generated by node 8 first travels to node 5 , and then the traffic is split into 2 paths: one is $5 \rightarrow 3 \rightarrow$ $6 \rightarrow 9 \rightarrow 10$ and the other path is $5 \rightarrow 2 \rightarrow 1 \rightarrow 10$. Figure $7(\mathrm{c})$ indicates that the traffic from node 7 to node 4 is via a single path, that is, $7 \rightarrow 1 \rightarrow 10 \rightarrow 9 \rightarrow 4$. Similarly, as shown in Figures 7(d) and 7(e), the routing path for traffic flow from node 5 to node 9 is $5 \rightarrow 3 \rightarrow 6 \rightarrow 9$, while the traffic flow from node 3 to node 1 is via the path $3 \rightarrow 5 \rightarrow 2 \rightarrow 1$. Finally, the traffic flow generated from node 2 travels through 2 paths, one is through $2 \rightarrow$ $5 \rightarrow 3 \rightarrow 6$, and the other is via $2 \rightarrow 1 \rightarrow 10 \rightarrow$ $9 \rightarrow 6$. 


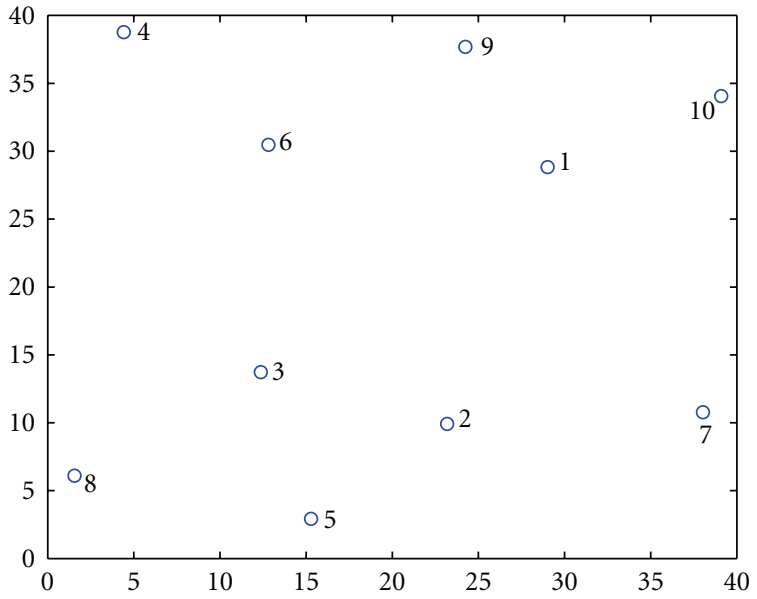

(a) Node location

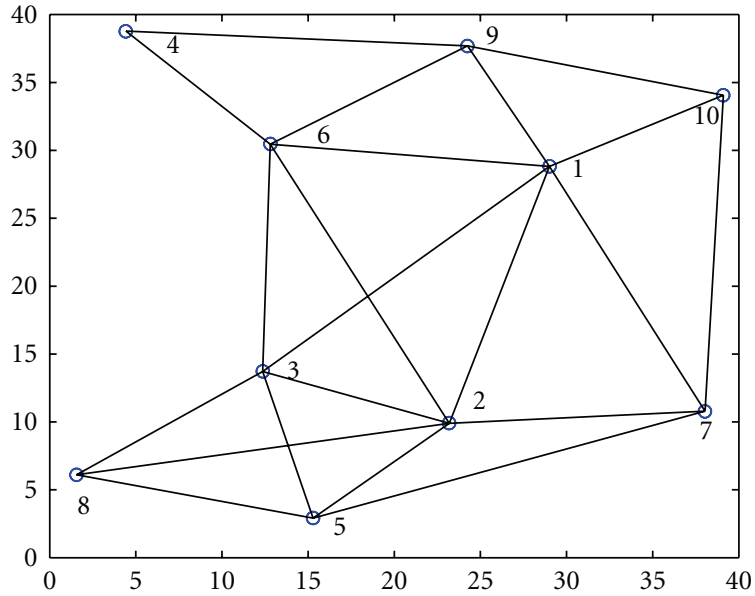

(b) Connectivity graph

FIGURE 6: Random topology.

TABLE 4: Throughput comparison for random topology.

\begin{tabular}{lcccc}
\hline $\begin{array}{l}\text { Experiment } \\
\text { index }\end{array}$ & $\begin{array}{c}\text { Global } \\
\text { optimal }\end{array}$ & $\begin{array}{c}\text { Relaxation } \\
\text { bound }\end{array}$ & Rounding & $\begin{array}{c}\text { Rounding \& } \\
\text { local } \\
\text { optimization }\end{array}$ \\
\hline 1 & 404.67 & 725.38 & 260.50 & 404.67 \\
2 & 508.28 & 860.37 & 504.81 & 504.81 \\
3 & 534.48 & 860.37 & 260.50 & 534.48 \\
4 & 183.67 & 183.67 & 154.17 & 183.67 \\
5 & 254.14 & 416.66 & 144.17 & 254.14 \\
6 & 247.78 & 422.54 & 123.89 & 247.78 \\
7 & 154.17 & 183.67 & 117.63 & 154.17 \\
8 & 152.76 & 183.67 & 101.84 & 152.76 \\
9 & 222.72 & 286.79 & 123.89 & 222.72 \\
10 & 177.44 & 286.79 & 123.89 & 177.44 \\
11 & 140.07 & 183.67 & 126.48 & 140.07 \\
12 & 130.25 & 183.67 & 123.89 & 130.25 \\
13 & 123.89 & 215.09 & 117.63 & 117.63 \\
14 & 148.48 & 183.67 & 94.86 & 148.48 \\
15 & 111.34 & 167.47 & 104.54 & 111.34 \\
\hline
\end{tabular}

\section{Conclusion}

In this paper, we consider a multihop multi-channel CR network. We present a cross-layer optimization framework by jointly designing the spectrum sharing and routing with the SINR constraints. Distinguished from the previous studies, we adopt a more realistic SINR model to capture the conflict relationships among the links, rather than using the Protocol Model. Our objective is to maximize the minimum end-to-end flow throughput, and our study addresses the following two cross-layer throughput optimization problem. (1) Given a set of secondary users with random but fixed location, and a set of traffic flows, what is the max-min achievable throughput? (2) To achieve the optimum, how to choose the set of active links, how to assign the channels
TABLE 5: Optimality ratio comparison for random topology.

\begin{tabular}{lccc}
\hline $\begin{array}{l}\text { Experiment } \\
\text { index }\end{array}$ & $\begin{array}{c}\text { Relaxation } \\
\text { bound }\end{array}$ & Rounding & $\begin{array}{c}\text { Rounding \& } \\
\text { local } \\
\text { optimization }\end{array}$ \\
\hline 1 & 1.79 & 0.64 & 1.00 \\
2 & 1.69 & 0.99 & 0.99 \\
3 & 1.61 & 0.49 & 1.00 \\
4 & 1.00 & 0.84 & 1.00 \\
5 & 1.64 & 0.57 & 1.00 \\
6 & 1.71 & 0.50 & 1.00 \\
7 & 1.19 & 0.76 & 1.00 \\
8 & 1.20 & 0.67 & 1.00 \\
9 & 1.29 & 0.56 & 1.00 \\
10 & 1.62 & 0.70 & 1.00 \\
11 & 1.31 & 0.90 & 1.00 \\
12 & 1.41 & 0.95 & 1.00 \\
13 & 1.74 & 0.95 & 0.95 \\
14 & 1.24 & 0.64 & 1.00 \\
15 & 1.50 & 0.94 & 1.00 \\
\hline
\end{tabular}

TABLE 6: Rate requirements of 5 sessions for random topology.

\begin{tabular}{lc}
\hline Source node & Destination node \\
\hline 8 & 10 \\
7 & 4 \\
5 & 9 \\
3 & 1 \\
2 & 6 \\
\hline
\end{tabular}

to each active link, and how to route the flows? We answer these questions via a formal mathematical formulation in the forms of mixed integer nonlinear programming (MINLP). Since the MINLP formulation is generally an NP-hard problem, we develop a heuristic method by solving a relaxation 


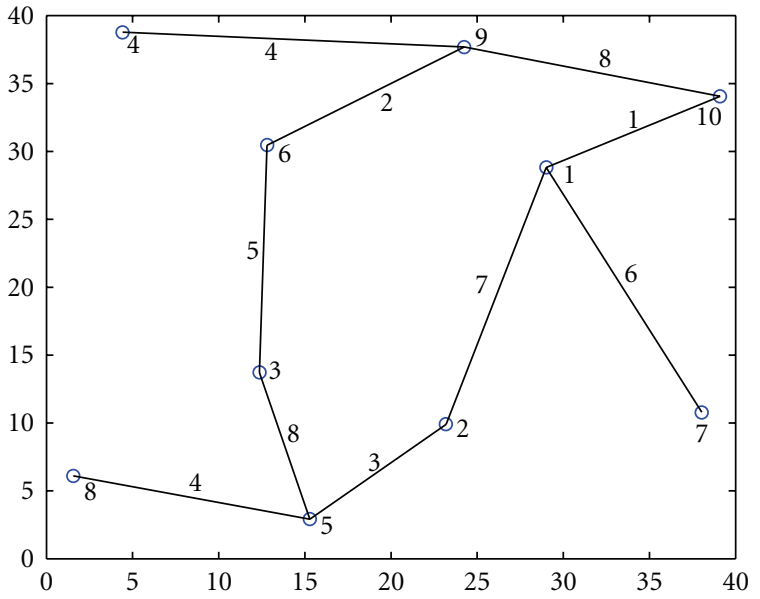

(a) Spectrum sharing

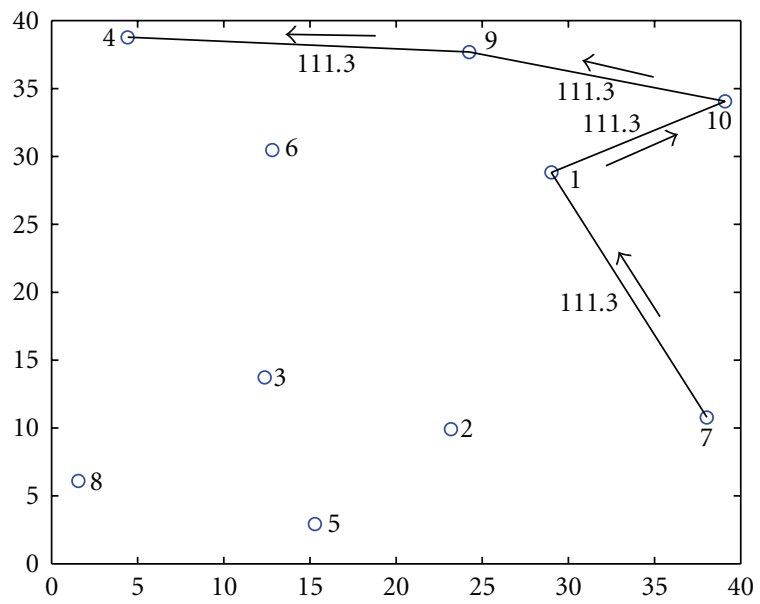

(c) Routing flow 2

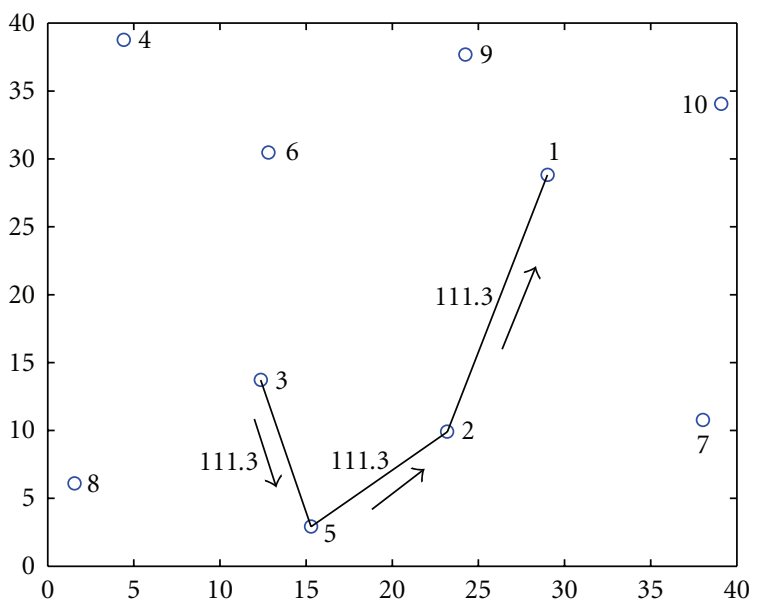

(e) Routing flow 4

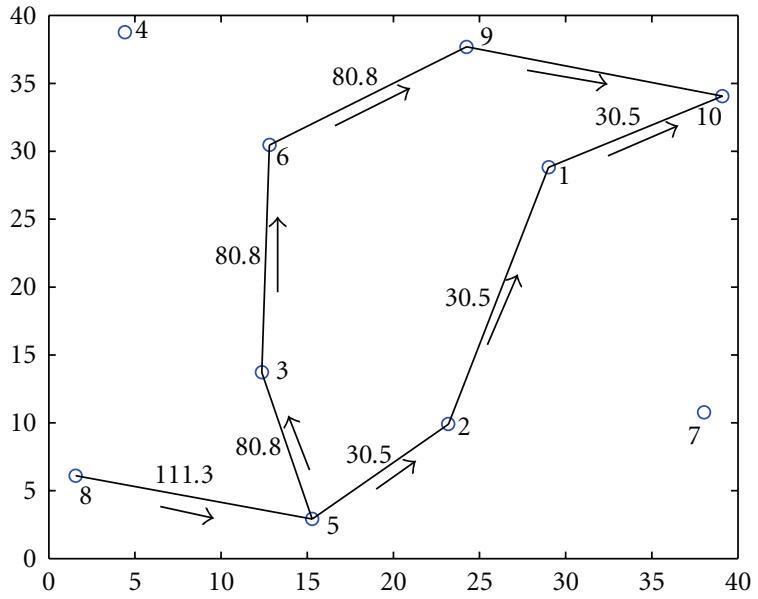

(b) Routing flow 1

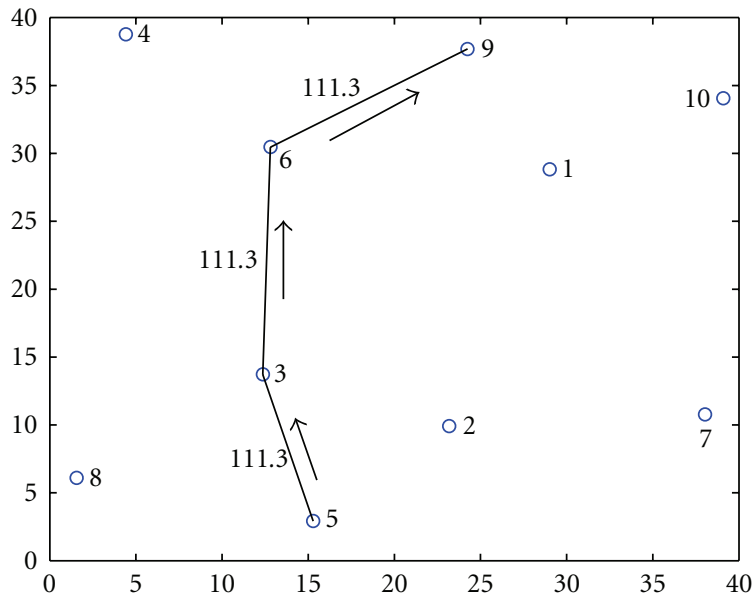

(d) Routing flow 3

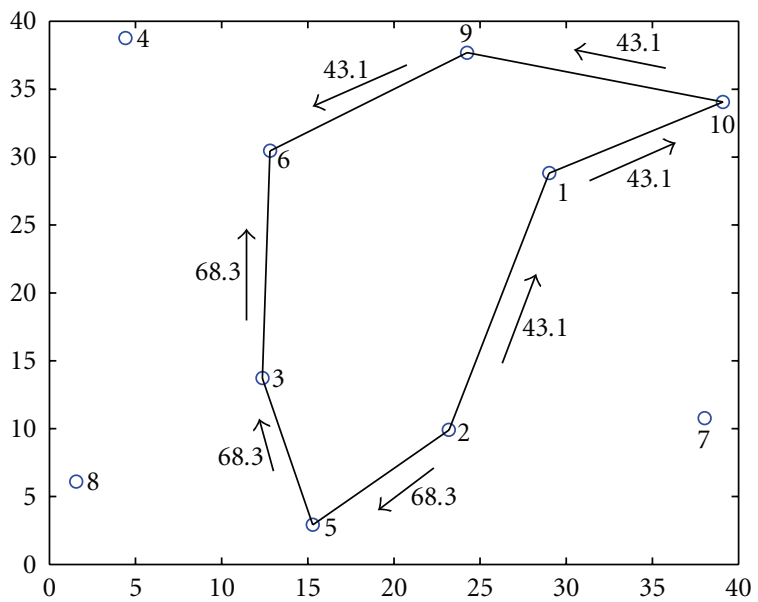

(f) Routing flow 5

FIGURE 7: Spectrum sharing and routing for random topology.

of the original problem, followed by rounding and simple local optimization. Simulation results show that the heuristic approach performs very well; that is, the solutions obtained by the heuristic are very close to the global optimum.
For the future work, we need to consider how to design a distributed algorithm for a multihop CR network. Since in reality, there may not exist a centralized server, and also, the available channels are highly dynamic, in such 


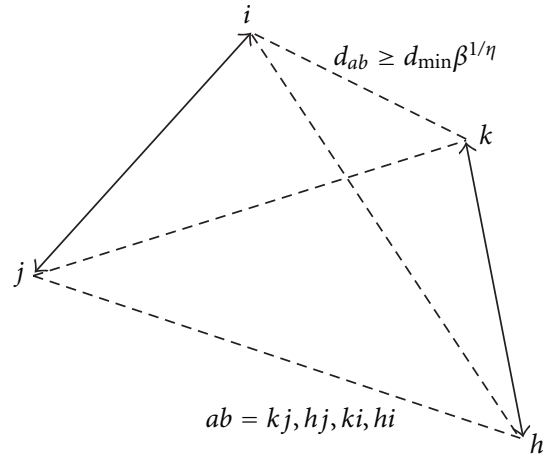

FIGURE 8: SINR model of interference.

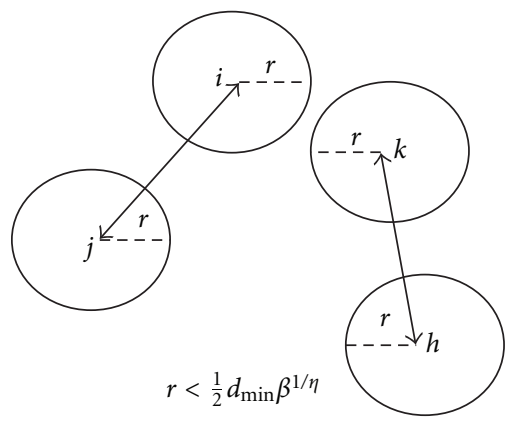

FIGURE 9: Disks of radius $r$ centered at transmitters and receivers are disjoint.

situation, how to choose the set of active links and how to allocate channels and route the flows to obtain the max-min achievable throughput is a highly desirable and challenging work.

\section{Appendix}

\section{Proof of Theorem 1}

Consider two links, say $e=(i, j)$ and $e^{\prime}=(k, h)$, using a same channel for simultaneous transmission. According to the SINR model, the transmission on links $e$ is successful if and only if

$$
\frac{P\left(d_{0} / d_{i j}\right)^{\eta}}{N+P\left(d_{0} / d_{a b}\right)^{\eta}} \geq \beta, \quad \text { for } a b=k j, h j, k i, h i .
$$

Then we find that $d_{a b}$ is bounded by $d_{i j} \beta^{1 / \eta}$, that is,

$$
d_{a b} \geq d_{i j} \beta^{1 / \eta}, \quad \text { for } a b=k j, h j, k i, h i \text {. }
$$

Suppose that the minimum distance of any two nodes is $d_{\min }$; we have

$$
d_{a b} \geq d_{\min } \beta^{1 / \eta}, \quad \text { for } a b=k j, h j, k i, h i .
$$

Thus, for links $e$ and $e^{\prime}$ to be independent, the distances $d_{k j}, d_{h j}, d_{k i}$, and $d_{h i}$ have to exceed $d_{\min } \beta^{1 / \eta}$ (see Figure 8 ). This is equivalent to embed the disjoint disks of radius $d_{\min } \beta^{1 / \eta} / 2$ centered at both transmitters and receivers, as shown in Figure 9. Different from the case of the unidirectional link $[16,25]$, for the bidirectional link both the transmitter and the receiver "consume" a "wireless footprint" of area, and the total consumed area of a bidirectional link is $2 \pi\left(d_{\min } \beta^{1 / \eta} / 2\right)^{2}$. Note that the total area of the domain is $L^{2}$ square meters. Thus, at most $2 L^{2} / \pi\left(d_{\min } \beta^{1 / \eta}\right)^{2}$ transmissions are simultaneously feasible. That is, the number of simultaneous transmissions on a same channel is upper bounded by $2 L^{2} / \pi\left(d_{\min } \beta^{1 / \eta}\right)^{2}$.

\section{References}

[1] J. Mitola III and G. Q. Maguire Jr., "Cognitive radio: making software radios more personal," IEEE Personal Communications, vol. 6, no. 4, pp. 13-18, 1999.

[2] S. Haykin, "Cognitive radio: brain-empowered wireless communications," IEEE Journal on Selected Areas in Communications, vol. 23, no. 2, pp. 201-220, 2005.

[3] I. F. Akyildiz, W.-Y. Lee, M. C. Vuran, and S. Mohanty, "NeXt generation/dynamic spectrum access/cognitive radio wireless networks: a survey," Computer Networks, vol. 50, no. 13, pp. 2127-2159, 2006.

[4] Y. T. Hou, Y. Shi, and H. D. Sherali, "Optimal spectrum sharing for multi-hop software defined radio networks," in Proceedings of the 26th IEEE International Conference on Computer Communications (INFOCOM '07), pp. 1-9, May 2007.

[5] Y. Shi and Y. T. Hou, "Optimal power control for multi-hop software defined radio networks," in Proceedings of the 26th IEEE International Conference on Computer Communications (INFOCOM '07), pp. 1694-1702, May 2007.

[6] Y. Shi and Y. T. Hou, "A distributed optimization algorithm for multi-hop cognitive radio networks," in Proceedings of the 27th IEEE International Conference on Computer Communications (INFOCOM '08), pp. 1966-1974, April 2008.

[7] M. Ma and D. H. K. Tsang, "Efficient spectrum sharing and power control in cognitive radio networks," in Proceedings of the 1st International Workshop on Cognitive Wireless Networks (CWNETS '07), 2007.

[8] M. Ma and D. H. K. Tsang, "Joint design of spectrum sharing and routing with channel heterogeneity in cognitive radio networks," Physical Communication, vol. 2, no. 1-2, pp. 127137, 2009.

[9] K. Jain, J. Padhye, V. N. Padmanabhan, and L. Qiu, "Impact of interference on multi-hop wireless netwrok performance," in Proceedings of the 9th Annual International Conference on Mobile Computing and Networking (MOBICOM '03), pp. 6680, September 2003.

[10] A. Iyer, C. Rosenberg, and A. Karnik, "What is the right model for wireless channel interference?" in Proceedings of the $3 \mathrm{rd}$ International Conference on Quality of Service in Heterogeneous Wired/Wireless Networks (QShine '06), vol. 191, Waterloo, Canada, August 2006.

[11] G. Brar, D. M. Blough, and P. Santi, "Computationally efficient scheduling with the physical interference model for throughput improvement in wireless mesh networks," in Proceedings of the 12th Annual International Conference on Mobile Computing and Networking (MOBICOM '06), pp. 213, September 2006.

[12] D. Chafekar, V. S. A. Kumar, M. V. Marathe, S. Parthasarathy, and A. Srinivasan, "Cross-layer latency minimization in wireless networks with SINR constraints," in Proceedings of the 8th 
ACM International Symposium on Mobile Ad Hoc Networking and Computing (MobiHoc'07), pp. 110-119, September 2007.

[13] A. Behzad and I. Rubin, "Optimum integrated link scheduling and power control for multihop wireless networks," IEEE Transactions on Vehicular Technology, vol. 56, no. 1, pp. 194205, 2007.

[14] LINDO Systems, LINGO: User's Guide, 2006.

[15] C. Xin, B. Xie, and C.-C. Shen, "A novel layered graph model for topology formation and routing in dynamic spectrum access networks," in Proceedings of the 1st IEEE International Symposium on New Frontiers in Dynamic Spectrum Access Networks (DySPAN '05), pp. 308-317, November 2005.

[16] S. Narayanaswamy, V. Kawadia, R. S. Sreenivas, and P. R. Kumar, "Power control in ad-hoc networks: theory, architecture, algorithm and implementation of the COMPOW protocol," in Proceedings of European Wireless, 2002.

[17] B. Hajek and G. Sasaki, "Link scheduling in polynomial time," IEEE Transactions on Information Theory, vol. 34, no. 5, pp. 910-917, 1988.

[18] G. Wang and N. Ansari, "Optimal broadcast scheduling in packet radio networks using mean field annealing," IEEE Journal on Selected Areas in Communications, vol. 15, no. 2, pp. 250-259, 1997.

[19] J. P. Monks, V. Bharghavan, and W. W. Hwu, "A power controlled multiple access protocol for wireless packet networks," in Proceedings of the 20th Annual Joint Conference on the IEEE Computer and Communications Societies (INFOCOM '01), pp. 219-228, April 2001.

[20] T. ElBatt and A. Ephremides, "Joint scheduling and power control for wireless ad-hoc networks," in Proceedings of the 21th Annual Joint Conference on the IEEE Computer and Communications Societies (INFOCOM '02), pp. 976-984, June 2002.

[21] A. Muqattash and M. Krunz, "Power controlled dual channel (PCDC) medium access protocol for wireless ad hoc networks," in Proceedings of the 22nd Annual Joint Conference on the IEEE Computer and Communications Societies (INFOCOM '03), pp. 470-480, April 2003.

[22] Y. Xi and E. M. Yeh, "Distributed algorithms for spectrum allocation, power control, routing, and congestion control in wireless networks," in Proceedings of the 8th ACM International Symposium on Mobile Ad Hoc Networking and Computing (MobiHoc '07), pp. 180-189, September 2007.

[23] P. Gupta and P. R. Kumar, "The capacity of wireless networks," IEEE Transactions on Information Theory, vol. 46, no. 2, pp. 388-404, 2000.

[24] L. Badia, A. Erta, L. Lenzini, and M. Zorzi, "A general interference-aware framework for joint routing and link scheduling in wireless mesh networks," IEEE Network, vol. 22, no. 1, pp. 32-38, 2008.

[25] A. Karnik, A. Iyer, and R. C. Rosenberg, "Throughputoptimal configuration of fixed wireless networks," IEEE/ACM Transactions on Networking, vol. 16, no. 5, pp. 1161-1174, 2008.

[26] A. Zymnis, S. Boyd, and D. Gorinevsky, "Relaxed maximum a posteriori fault identification," Signal Processing, vol. 89, no. 6, pp. 989-999, 2009. 

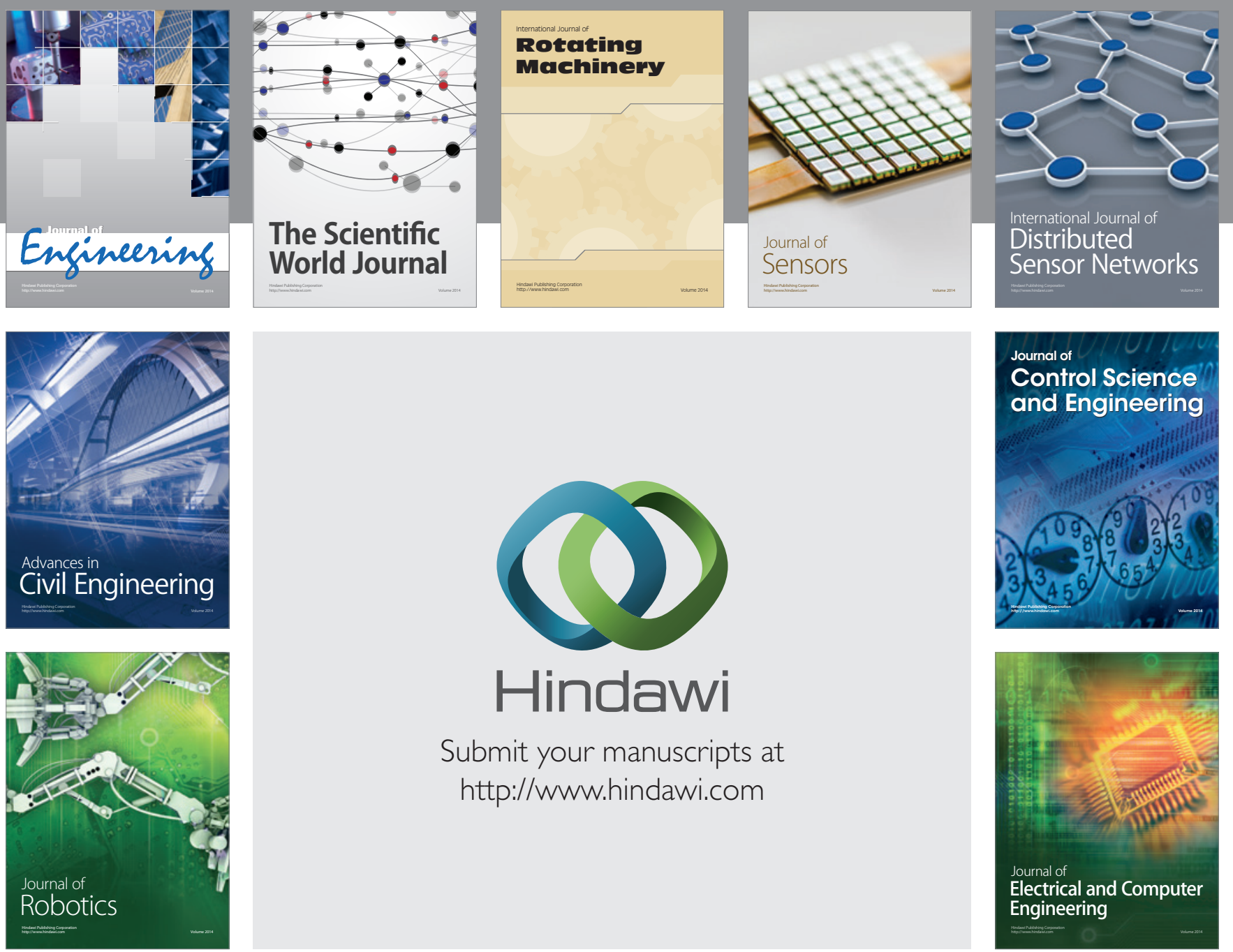

Submit your manuscripts at

http://www.hindawi.com
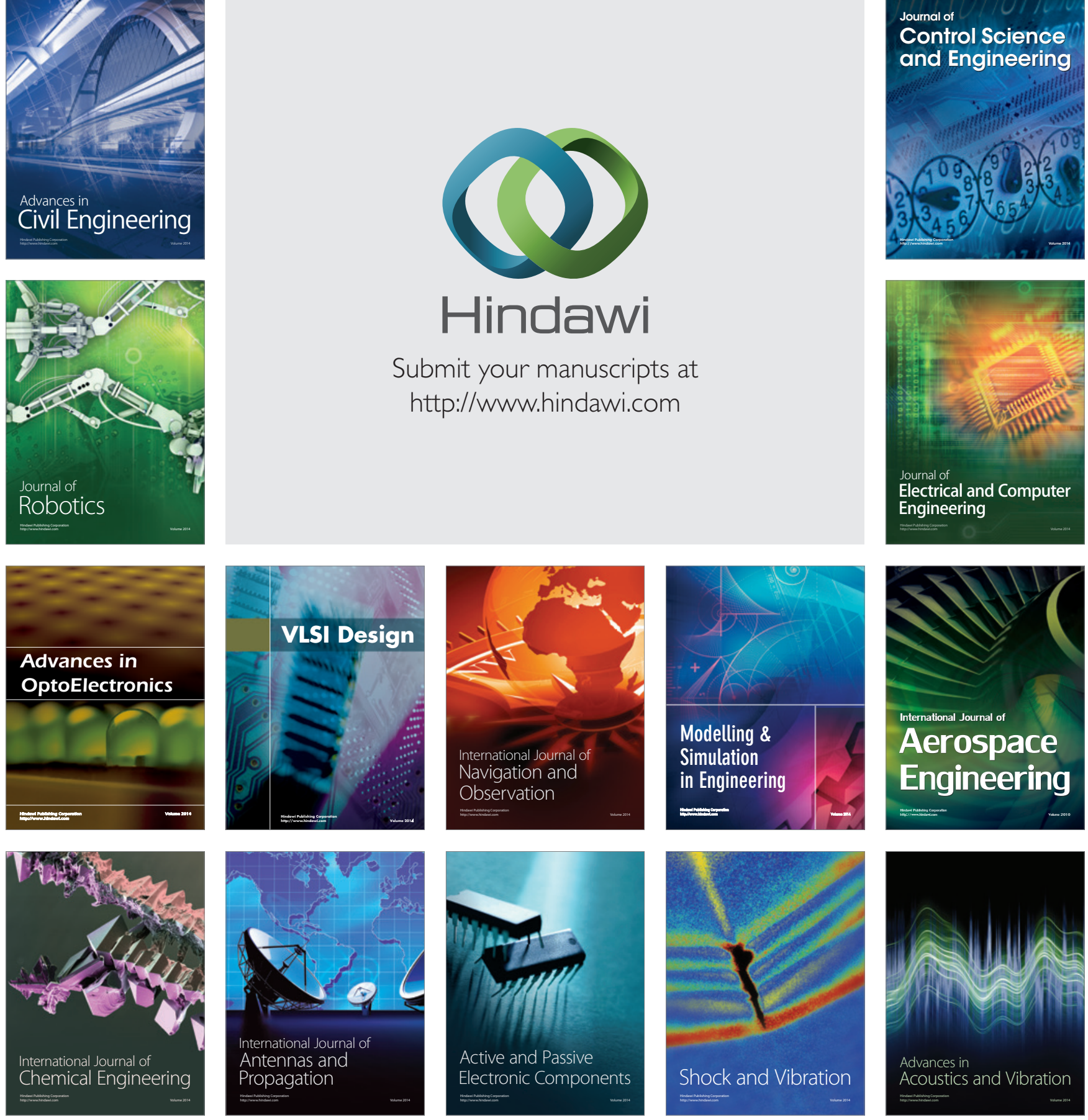